\title{
Chapter 8 \\ LEAP-2017 Simulation Exercise: Overview of Guidelines for the Element Test Simulations
}

\author{
Majid T. Manzari, Mohamed El Ghoraiby, Bruce L. Kutter, \\ and Mourad Zeghal
}

\begin{abstract}
This paper summarizes the guidelines provided to the numerical simulation/prediction teams that participated in the LEAP-2017 prediction exercise. These guidelines are developed for the Phase 1 of the simulations that focused on the use of cyclic triaxial element tests for calibration of constitutive models that the participating teams used in their numerical simulations/predictions.
\end{abstract}

\subsection{Introduction}

LEAP-2017 is a collaboration within the framework of the Liquefaction Experiments and Analysis Projects to produce high-quality centrifuge data of a mild slope and validate constitutive models and numerical tools used in soil liquefaction analysis. This collaboration involved a simulation exercise which provided an opportunity to evaluate the capabilities and limitations of a large number of commonly used constitutive and numerical modeling techniques for liquefaction analysis. A number of simulation teams from the geotechnical engineering community across the world participated in modeling a selection of the performed centrifuge experiments. The simulation exercise consisted of constitutive model calibration, Type-B prediction, and Type-C prediction.

\footnotetext{
M. T. Manzari $(\bowtie)$

Department of Civil and Environmental Engineering, George Washington University,

Washington, DC, USA

e-mail:manzari@gwu.edu

M. El Ghoraiby

The George Washington University, Washington, DC, USA

B. L. Kutter

Department of Civil and Environmental Engineering, University of California, Davis, CA, USA

M. Zeghal

Department of Civil and Environmental Engineering, Rensselaer Polytechnic Institute, Troy, NY, USA
} 
A series of laboratory experiments were first conducted on the designated soil for LEAP (Ottawa F65 sand) and made available to the simulation teams (hereafter referred to as predictors) for calibration of the constitutive models that were later used in Phase II and Phase III of the simulation exercise (i.e., Type-B and Type-C simulations). A Type-B simulation consists of analyzing the centrifuge experiments without any knowledge of the experiment results while the base excitation and achieved soil density are provided. For the Type-C prediction, the predictors were provided with the experimental results.

An outline of the essential elements of the calibration phase of the simulation exercise is presented in this paper. Each simulation team was requested to submit a detailed report discussing the process followed in the calibration of their selected constitutive model. The submitted report by each team was intended to provide a description of the constitutive model used by the team as well as the method used for calibration.

The submitted simulation results were requested to follow specific formats to facilitate future assessment and comparison efforts. The formatting of the data files is described in this report along with a template of the results. Matlab-based visualization tools were also provided to the numerical simulation teams for plotting the available cyclic triaxial experiments and the simulation results before submittal.

\subsection{Soil Characterization and Element Tests}

\subsubsection{LEAP-2017 Tests}

The soil characterization and element tests on Ottawa F65 sand are discussed in El Ghoraiby et al. (2017, 2018). The soil characterization tests conducted included particle-size distribution, specific gravity, and permeability tests. The results of these tests are reported for five different batches of Ottawa F65 sand. The means and standard deviations of the measured results were also presented. A series of cyclic stress-controlled triaxial tests conducted by El Ghoraiby et al. (2017) were also made available to the prediction teams for three different soil densities. Details of sample preparation using "Constant Height Dry Pluviation" technique are presented in El Ghoraiby et al. (2017). Tables 8.1, 8.2, and 8.3 show a summary of the triaxial tests conducted for LEAP-2017 project.

Figure 8.1 shows the liquefaction strength curves for the three series of cyclic stress-controlled triaxial tests.

The element tests of LEAP-2017 project supplemented an earlier series of tests conducted by Vasko (2015) which focused on monotonic and cyclic straincontrolled tests. The results of the tests reported in Vasko (2015) were also made available to the numerical simulation teams via the NEEShub data repository and are now available on DesignSafe (Vasko et al. 2018).

The data resulted from the cyclic stress-controlled tests were saved as tab delimited text files in the folder named "CyclicStressControlledTests". The file 
Table 8.1 Summary of cyclic stress-controlled triaxial tests on specimens with $e_{\mathrm{o}}=0.515$

\begin{tabular}{l|l|l|l|l}
\hline \multicolumn{1}{l}{$e_{\mathrm{c}}=0.515$} & $-\rho_{\mathrm{d}}=1744 \mathrm{~kg} / \mathrm{m}^{3}$ \\
\hline Date & File name & $e_{\mathrm{c}}$ & CSR & No. of cycles to 2.5\% S.A. \\
\hline 10/10/2016 & eo_0_515_po_100_csr_0_6.txt & 0.515 & 0.6 & 14 \\
\hline 10/6/2016 & eo_0_515_po_100_csr_0_5.txt & 0.522 & 0.5 & 17 \\
\hline 10/7/2016 & eo_0_515_po_100_csr_0_45.txt & 0.512 & 0.45 & 19 \\
\hline 9/16/2016 & eo_0_515_po_100_csr_0_375.txt & 0.515 & 0.375 & 26 \\
\hline 9/27/2016 & eo_0_515_po_100_csr_0_365.txt & 0.514 & 0.365 & 29 \\
\hline 9/30/2016 & eo_0_515_po_100_csr_0_325.txt & 0.507 & 0.325 & 41 \\
\hline 9/22/2016 & eo_0_515_po_100_csr_0_315.txt & 0.514 & 0.315 & 46 \\
\hline 9/28/2016 & eo_0_515_po_100_csr_0_3.txt & 0.516 & 0.3 & 48 \\
\hline 9/13/2016 & eo_0_515_po_100_csr_0_275.txt & 0.517 & 0.275 & 60 \\
\hline 9/29/2016 & eo_0_515_po_100_csr_0_265.txt & 0.515 & 0.265 & 70 \\
\hline 10/12/2016 & eo_0_515_po_100_csr_0_225.txt & 0.513 & 0.225 & 191
\end{tabular}

Table 8.2 Summary of cyclic stress-controlled triaxial tests with $e_{\mathrm{o}}=0.542$

\begin{tabular}{l|l|l|l|l}
\hline \multicolumn{1}{c}{$=$} \\
\hline Date & File name & $e_{\mathrm{c}}$ & CSR & No. of cycles to 2.5\% S.A. \\
\hline $11 / 20 / 2016$ & eo_0_542_po_100_csr_0_28.txt & 0.55 & 0.28 & 16 \\
\hline $11 / 18 / 2016$ & eo_0_542_po_100_csr_0_24.txt & 0.54 & 0.24 & 18 \\
\hline $11 / 16 / 2016$ & eo_0_542_po_100_csr_0_22.txt & 0.535 & 0.22 & 22 \\
\hline $11 / 16 / 2016$ & eo_0_542_po_100_csr_0_21.txt & 0.538 & 0.21 & 28 \\
\hline $11 / 16 / 2016$ & eo_0_542_po_100_csr_0_2.txt & 0.55 & 0.2 & 41 \\
\hline $11 / 21 / 2016$ & eo_0_542_po_100_csr_0_19.txt & 0.538 & 0.19 & 50
\end{tabular}

Table 8.3 Summary of cyclic stress-controlled triaxial tests $e_{\mathrm{o}}=0.585$

\begin{tabular}{|c|c|c|c|c|}
\hline \multicolumn{5}{|c|}{$e_{\mathrm{c}}=0.585-\rho_{\mathrm{d}}=1666 \mathrm{~kg} / \mathrm{m}^{3}$} \\
\hline Date & File name & $e_{\mathrm{c}}$ & CSR & No. of cycles to $2.5 \%$ S.A. \\
\hline $11 / 10 / 2016$ & eo_0_585_po_100_csr_0_2.txt & 0.581 & 0.2 & 9 \\
\hline $11 / 1 / 2016$ & eo_0_585_po_100_csr_0_17.txt & 0.584 & 0.17 & 15 \\
\hline $11 / 2 / 2016$ & eo_0_585_po_100_csr_0_16.txt & 0.587 & 0.16 & 17 \\
\hline $11 / 4 / 2016$ & eo_0_585_po_100_csr_0_14.txt & 0.575 & 0.14 & 33 \\
\hline $11 / 7 / 2016$ & eo_0_585_po_100_csr_0_12.txt & 0.598 & 0.12 & 59 \\
\hline $11 / 14 / 2016$ & eo_0_585_po_100_csr_0_1.txt & 0.583 & 0.1 & 188 \\
\hline
\end{tabular}

names are listed in Tables 8.1, 8.2, and 8.3. Each file is composed of six columns. The first column is time in minutes. The second and third columns show the axial and volumetric strains, respectively, in \%. The fourth, fifth, and sixth columns, respectively, show $\sigma_{3}^{\prime}, \sigma_{3}$, and $\sigma_{\mathrm{d}}$, all in $\mathrm{kPa}$. This folder and its corresponding files is archived in DesignSafe.

To graph the test results reported in each file, a Matlab script (PlotExpResults.m) is provided in the same folder. The script allows the user to plot time histories of axial strain, deviatoric stress, and mean effective stress. PlotExpResults.m also provides plots of deviatoric stress vs. axial strain, effective stress path, as well as excess pore pressure ratio and mean effective stress vs. axial strain. Summery plots 


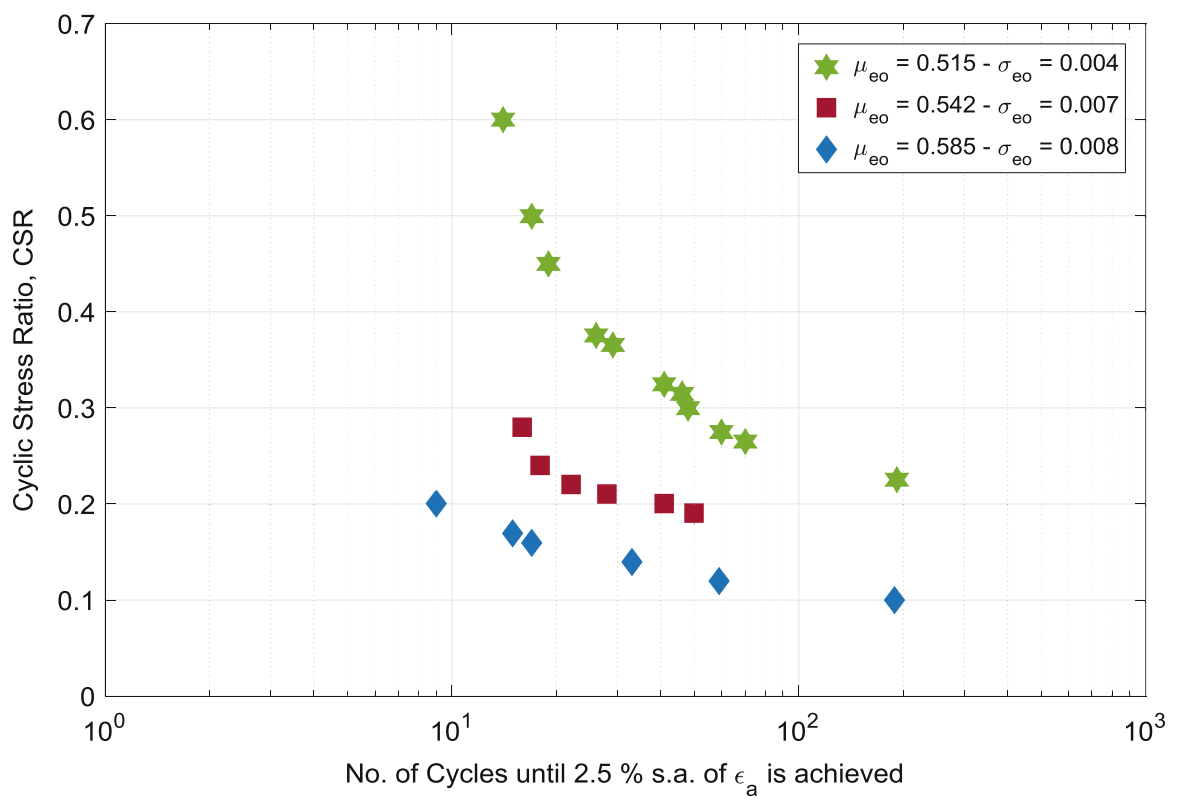

Fig. 8.1 Liquefaction strength curves obtained from cyclic stress-controlled triaxial tests on Ottawa F65 sand (El Ghoraiby et al. 2017)

of the cyclic stress-controlled tests are presented in the appendices A-C of "LEAP2017 soil characterization and element tests of Ottawa F65 sand" (El Ghoraiby et al. 2017).

\subsubsection{Additional Available Element Tests on Ottawa Sand}

Additional experimental data on Ottawa F65 was also available and could be used by the simulation teams for their model calibration purpose, if needed. The simulation teams were made aware of the facts that the sample preparation techniques (which influence the fabrics of the prepared samples) used in these tests may be different from the cyclic stress-controlled triaxial tests performed for LEAP-2017.

The experimental data that was available during the planning phase of LEAP and for LEAP-2015 prediction exercise could be accessed at the following link (Vasko et al. 2018):

https://doi.org/10.17603/DS2TH7Q.

The data accessible via the above link consists of two sets of experiments performed by different researchers.

The first set of experiments consists of triaxial experiments performed by Andrew Vasko at the George Washington University (Vasko 2015). The soil samples in these 
experiments were prepared using dry pluviation with minor tapping on the mold to achieve the desired density. For additional information the reader is referred to Vasko (2015).

The second set of experiments is the direct simple shear experiments (monotonic and cyclic) performed by Ana Maria Parra Bastidas at the University of California, Davis. These experiments were performed using dry funnel deposition (for loose samples) and air pluviation (for dense samples) methods. For additional information the reader is referred to Bastidas (2016) and Bastidas et al. (2017).

If the simulation teams decided to rely on the additional experiments discussed above, they were asked to clearly state that in the calibration report. The specific additional tests used should be clearly identified in the report. Despite the dataset used for calibration, the numerical simulation teams were asked to simulate the cyclic triaxial tests presented in El Ghoraiby et al. (2017) using the final set of calibration parameters.

\subsection{Model Calibration Report by Simulation Teams}

Each simulation team was requested to submit a calibration report discussing the process that was followed in the calibration process. The calibration report was intended to cover the essential features of the constitutive model, the final model parameters, the calibration philosophy, and any assumptions used in the calibration process. It also presented a comparison of the liquefaction strength curves obtained from the model simulation against the results obtained from cyclic stress-controlled tests (Fig. 8.1).

\subsubsection{Model Description}

Each simulation team was asked to briefly describe the constitutive model used in their element test simulation in the calibration report. If the model in the exact form used in calibrations was published elsewhere, a reference to the published article was included in the report. The reports were also to cover the main features of the model including the framework on which the model formulation was based (classical, bounding surface, two-surface or multi-surface plasticity, etc.), a description of the model internal variables and their functionality within the model, and any additional modifications to the original model formulation. Furthermore, the model description was to include the type of analysis platform on which the constitutive model was implemented (FEM, FDM, Mesh-free, etc.) and the integration scheme used in the implementation (implicit, explicit, and semi-implicit). 


\subsubsection{Model Parameters}

The calibration reports had to list the values of all the model parameters obtained from the calibration process. The list of parameters included the values of any parameter that is kept at a default value. If more than one set of parameters were used for different soil densities, separate lists of model parameters for each case and the rationale for using more than one set of parameters were to be provided. If the model parameters were obtained by using the additional element test data (other than the cyclic triaxial stress-controlled tests shown in Fig. 8.1), the numerical simulation teams were asked to clearly state that in the calibration report.

\subsubsection{Calibration Method}

The numerical simulation teams were asked to discuss the approach used in the calibration of the constitutive model parameters and to explain how the element tests (cyclic triaxial and cyclic direct simple shear) were simulated. For example, the calibration reports were expected to include a discussion on how the internal variables were initialized for each test. The initial values assigned to the internal variables were also to be reported for each simulated test.

\subsubsection{Liquefaction Strength Curves}

Each calibration report was to include a plot comparing the simulated liquefaction strength curves with the results of cyclic stress-controlled triaxial tests reported by El Ghoraiby et al. (2017). The simulation results were also listed in a table which reported the number of cycles until $2.5 \%$ single amplitude axial strain is achieved for each test.

\subsection{Simulation Results}

In addition to the report on model calibration, the results of the simulation of the cyclic triaxial tests were asked to be reported in separate files with the formats discussed below. 


\subsubsection{Results Data Files}

The results files were requested to be submitted as tab delimited text files. The files were designated to be placed in a folder called "calibration". The number of cycles until a $2.5 \%$ single amplitude axial strain is achieved was to be reported in a separate file for each density. The file names were to be labeled "eo_0_515_csr_nocyc.txt", “eo_0_542_csr_nocyc.txt", and "eo_0_585_csr_nocyc.txt" for $e_{\mathrm{o}}$ of $0.515,0.542$, and 0.585 , respectively. Each file was composed of a table of three columns. The first column was the initial void ratio of the soil before the shearing phase, the second column was the cyclic stress ratio, and the third column was the number of cycles until a $2.5 \%$ single amplitude axial strain is achieved.

The simulation results for each cyclic triaxial test were to be reported in a $t a b$ delimited text file with the same name as the simulation performed. The file was composed of a three-column table. The first column was the axial strain, $\varepsilon_{\mathrm{a}}$; the second column was the deviatoric stress, $\sigma_{\mathrm{d}}$; and the third column was the confining effective stress, $\sigma_{3}^{\prime}$. The axial strain was reported in $\%$, while the stresses were reported in $\mathrm{kPa}$. Although simulations could have been run with variable time steps, the submitted results were asked to be reported with a constant time step.

The simulation results for cyclic direct simple shear tests were also reported in $t a b$ delimited text file with the same name as the simulation performed. The file was composed of a three-column table. The first column was the shear strain, $\varepsilon_{\mathrm{a}}$, the second column was the shear stress, $\tau$, and the third column was the vertical effective stress, $\sigma_{\mathrm{v}}^{\prime}$. The shear strain was reported in $\%$, while the stresses were reported in $\mathrm{kPa}$. Although simulations could have been run with variable time steps, the submitted results were asked to be reported with a constant time step.

\subsubsection{Matlab Scripts}

A series of Matlab scripts were provided to the numerical simulations teams so that they can confirm the format consistency of the calibration results files. The first Matlab script was called "LiqStrengthCurve.m". This file was intended to load the tab delimited text files of the CSR vs. No. of Cycles and plot the liquefaction strength curve, where the simulation results were plotted against the experiment results. This file was provided to the simulation teams to ensure that the submitted simulation files conform to the specified format and facilitate future comparisons of the simulation with experimental data.

The second Matlab script was called "SimResultsPlot.m". The script loads the results file of the simulations of the cyclic stress-controlled triaxial tests. It plots the results in terms of a deviatoric stress vs. axial strain and deviatoric stress vs. mean effective stress. These scripts are provided in El Ghoraiby et al. (2018). 


\subsection{Concluding Remarks}

The predictor teams were provided with the calibration data and requested to submit a detailed report by June 1,2017. The calibration report and all the simulation data files were asked to be uploaded in the designated dropbox folder created for each simulation team. A summary of the calibration efforts and the results reported by each numerical simulation team are provided in Manzari et al. (2019).

\section{References}

Bastidas, A. M. P. (2016). Ottawa F-65 Sand Characterization. PhD Dissertation, University of California, Davis.

Bastidas, A. M. P, Boulanger, R. W., Carey, T., \& DeJong, J. (2017). Ottawa F-65 Sand Data from Ana Maria Parra Bastidas. https://datacenterhub.org/resources/ottawa_f_65. https://doi.org/10. 17603/DS2MW2R

El Ghoraiby, M. A., Park, H., \& Manzari, M. (2017). LEAP 2017: Soil Characterization and Element Tests for Ottawa F65 Sand. Washington, DC: The George Washington University.

El Ghoraiby, M. A., Park, H., \& Manzari, M. (2018). LEAP-2017 GWU Laboratory Tests. DesignSafe-CI, Dataset. https://doi.org/10.17603/DS2210X.

Manzari, M. T., El Ghoraiby, M. A., Zeghal, M., Kutter, B. L., Arduino, P., Barrero, A. R., Bilotta, E., Chen, L., Chen, R., Chiaradonna, A., Elgamal, A. E., Fasano, G., Fukutake, K., Fuentes, W., Ghofrani, A., Ichii, K., Kiriyama, K., Lascarro, C., Mercado, V., Montgomery, J., Ozutsumi, O., Qiu, Z., Taiebat, M., Travasarou, T., Tsiaousi, D., Ueda, K., Ugalde, J., Wada, T., Wang, R., Yang, M., Zhang, J.-M., \& Ziotopoulou, K. (2019). LEAP-2017 simulation exercise: Calibration of constitutive models and simulation of the element tests. In B. Kutter et al. (Eds.), Model tests and numerical simulations of liquefaction and lateral spreading: LEAP-UCD-2017. New York: Springer.

Vasko, A. (2015). An Investigation into the Behavior of Ottawa Sand through Monotonic and Cyclic Shear Tests. Masters Thesis, The George Washington University.

Vasko, A., El Ghoraiby, M., \& Manzari, M. (2018). LEAP-GWU-2015 Laboratory Tests. DesignSafe-CI, Dataset. https://doi.org/10.17603/DS2TH7Q.

Open Access This chapter is licensed under the terms of the Creative Commons Attribution 4.0 International Licence (http://creativecommons.org/licenses/by/4.0/), which permits use, sharing, adaptation, distribution and reproduction in any medium or format, as long as you give appropriate credit to the original author(s) and the source, provide a link to the Creative Commons licence and indicate if changes were made.

The images or other third party material in this chapter are included in the chapter's Creative Commons license, unless indicated otherwise in a credit line to the material. If material is not included in the chapter's Creative Commons licence and your intended use is not permitted by statutory regulation or exceeds the permitted use, you will need to obtain permission directly from the copyright holder.

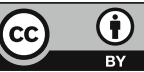

